\title{
Método de síntesis de nanopartículas de plata adaptable a laboratorios de docencia relacionado con la nanotecnología
}

\author{
Fernando M. Martinez, * Edgar Zuñiga G., * \\ Ana Karen Sanchez Lafarga*
}

\begin{abstract}
REsUmen: El auge de la nanociencia y la nanotecnología ha llevado al surgimiento de cursos formales especializados en este tema a nivel de enseñanza superior. Algunas universidades en México ya cuentan con asignaturas de naotecnología a nivel licenciatura, y para poder atraer estudiantes a esta área, es necesario motivarlos desde la enseñanza media a través de demostraciones experimentales de las propiedades de la materia en escala nanométrica.

Este trabajo presenta un método sencillo de síntesis de nanopartículas de plata diseñado con reactivos disponibles en cualquier laboratorio de docencia. Los resultados de este experimento demuestran que la plata en esta escala presenta diferente coloración a la conocida comúnmente en escala macroscópica, este cambio es un efecto de la resonancia del plasmón de superficie en nanopartículas metálicas.
\end{abstract}

PALABRAS CLAVE: nanopartículas, plata, plasmón, nanotecnología, enseñanza.

ABSTRACT: Nanoscience and nanotechnology development has led to the emergence of specialized courses on this subject at higher educational level. Some universities in Mexico already have courses at the undergraduate level, and in order to attract students to this area, it seems required to motivate them starting from middle school through experimental demonstrations of matter properties at the nanoscale.

This paper presents a simple method for synthesis of silver nanoparticles designed with reagents usually available in any mid-school laboratory. The results of this experiment demonstrate that silver at this scale has different coloration to the commonly known macroscopic scale. Such change is an effect of surface plasmon resonance in metal nanoparticles.

KEYWORDS: nanoparticles, silver, plasmon, nanotechnology, teaching.

\section{INTRODUCCIÓN}

La plata es un elemento metálico muy utilizado tradicionalmente en joyería, electrónica, electroquímica, ornamentación, etc.; esto permite que las propiedades de este metal en su forma macroscópica sean bien conocidas por la mayoría de los estudiantes; sin embargo, muy pocos conocen la capacidad de éstas para cambiar de manera apreciable cuando las dimensiones de las partículas de este metal están en el orden de los nanómetros (Chhatre, Solasa, Sakle, Thaokar y Mehra, 2012; Frank, Cathcart, Maly y Kitaev, 2010). Por esta razón, se considera importante mostrar a estudiantes

\footnotetext{
* Posgrado en Ciencias en Química. Departamento de Química. Universidad de Guadalajara. Boulevard Marcelino García Barragán 1421, CP 44430. Guadalajara, Jalisco, México.

<fermtzm@gmail.com>, <edgarzu_12@hotmail.com>, <karen.lafarga@hotmail.com>

Tel (33) 1378-5900 ext 27529.
} 
de preparatoria y licenciatura, cómo, a través de una práctica experimental, la plata puede tener diferentes propiedades.

Hasta ahora, algunos investigadores han propuesto algunas prácticas sencillas de laboratorio que pueden ser llevadas a cabo por estudiantes de licenciatura (Chhatre et al., 2012; Frank et al., 2010), el único inconveniente de estas prácticas es que se requiere de equipo sofisticado en cual no todas las universidades del mundo tienen a su disposición, y menos aún con accesibilidad para los estudiantes principiantes. Este hecho nos motiva diseñar un método de síntesis de nanopartículas de plata que pueda realizarse en prácticamente cualquier laboratorio de enseñanza superior y media superior.

Las nanopartículas de plata han atraído la atención debido a que dependiendo del tamaño o forma presentan las propiedades diferenciadas. En la antigüedad ya se empleaban las nanopartículas de plata y de algunos otros metales como oro, fungiendo éstas como pigmentos decorativos en artesanías, tiñendo vidrio o cerámica (Vankar y Shukla, 2011). En la actualidad, se ha logrado aprovechar en distintas áreas industriales y comerciales como bactericidas, sensores o incluso en la industria textil, debido a las diferentes coloraciones que puede presentar la plata en función de su forma y tamaño nanométrico (An, Zhu y Zhu, 2013).

Entre las propiedades características de la plata nanométrica, están las derivadas de la resonancia plasmónica de superficie (conocida por sus siglas en inglés: SPR, surface plasmon resonance). El fenómeno de SPR ocurre porque los electrones en la superficie de una nanopartícula metálica oscilan al interactuar con una onda electromagnética y se induce un momento dipolar sobre la partícula en un intervalo de tiempo (en la figura 1 se muestra cómo las cargas de electrones oscilan y se acumulan en los extremos). Cuando la componente eléctrica (línea negra) de la onda electromagnética (de un haz de luz visible) que incide sobre la nanopartícula oscila a la misma frecuencia que los electrones de ésta, ocurre el fenómeno de resonancia de plasmón de superficie.

Metales como $\mathrm{Au}, \mathrm{Ag}, \mathrm{Cu}, \mathrm{y}$ los metales alcalinos que poseen electrones libres muestran plasmón de resonancia en el espectro visible, dando lugar a colores no observados en los mismos materiales a escala macrométrica. Por ejemplo, las nanopartículas de plata y oro muestran una intensa SPR principalmente en los interva-

FIGURA 1. Representación del efecto oscilante de los electrones de superficie en nanopartículas metálicas y su interacción resonante con la luz (línea negra).

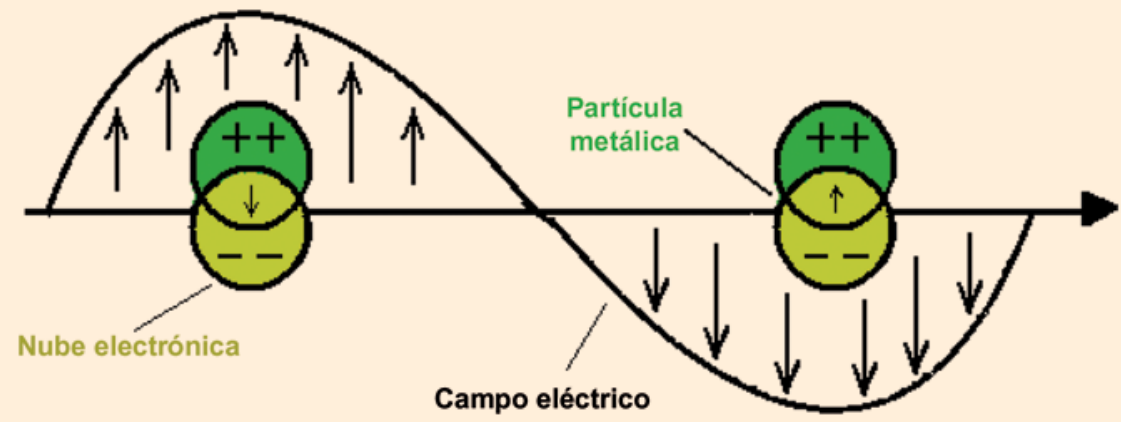


FIGURA 2. Espectros de UV-vis de soluciones de nanopartículas de plata con diferente radio. (Tang et al., 2013a).

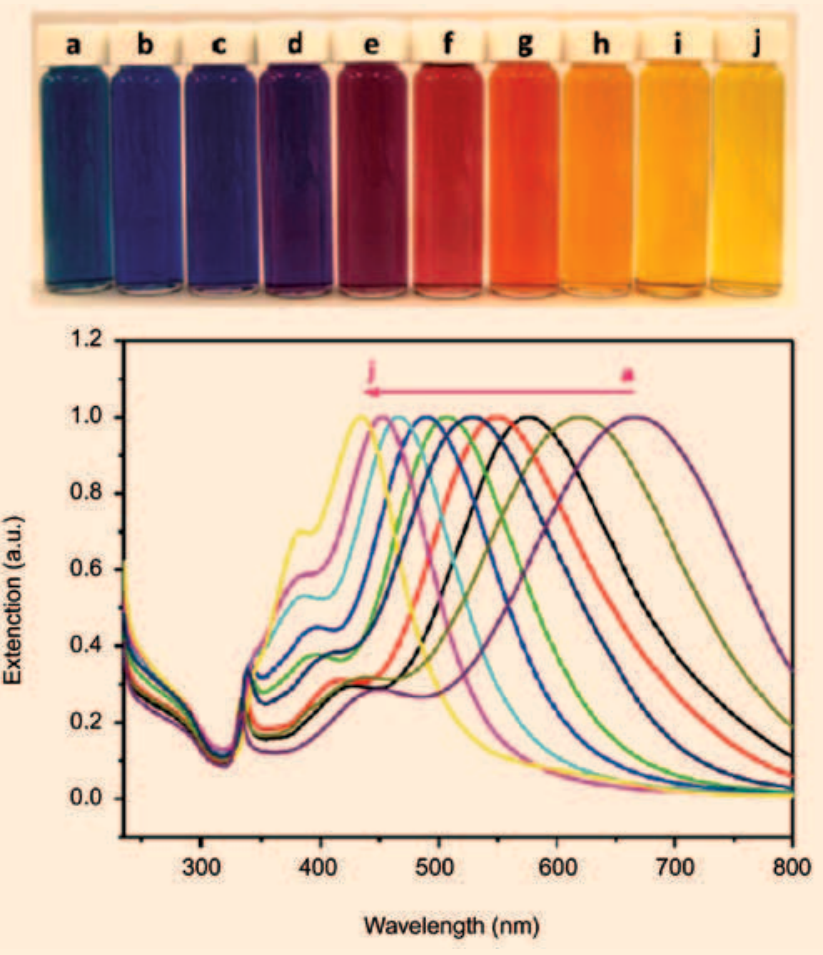

los de longitud de onda de 410-420 nm y 520-530 nm, respectivamente. La SPR y, por lo tanto, algunas propiedades ópticas de las nanopartículas dependen en gran medida de tamaño, forma, composición, índices de refracción del metal, el medio circundante, la presencia de especies adsorbidas y la distancia media entre nanopartículas vecinas (Chhatre et al., 2012; Frank et al., 2010; Vasileva, Donkova, Karadjova y Dushkin, 2011).

En la literatura se puede encontrar una diversidad de espectros de SPR de nanopartículas de plata en la región visible y las coloraciones que van del amarillo al azul (como se muestra en la figura 2). Por esto, para observar el fenómeno de la SPR de nanopartículas de plata en un laboratorio de docencia sólo se necesita un espectrómetro UV-vis como apoyo o en su defecto el ojo humano en aquellos laboratorios donde no se cuente con ese instrumento.

Por lo tanto, la propuesta de nuestro experimento es preparar nanopartículas de plata con reactivos de fácil acceso para cualquier laboratorio de enseñanza media y superior y que los alumnos puedan apreciar que la plata presenta una coloración diferente a la que convencionalmente presenta en tamaño macroscópico. 


\section{Metodología}

Se utilizaron los siguientes reactivos: ácido ascórbico (Productos Químicos Monterrey, $\mathrm{C}_{6} \mathrm{H}_{7} \mathrm{O}_{5} \mathrm{OH}, 99.9 \% \cdot$ ), citrato de potasio (Jalmek, $\mathrm{C}_{6} \mathrm{H}_{5} \mathrm{~K}_{3} \mathrm{O}_{7} \cdot \mathrm{H}_{2} \mathrm{O}, 99 \%$ ), hidróxido de sodio (Fermont, $\mathrm{NaOH}, 98.7 \%$ ) y nitrato de plata (Golden Bell, $\mathrm{AgNO}_{3}$, solución estándar).

Las nanopartículas de plata se forman por la reducción de la plata $\mathrm{Ag}^{+}$en la forma de nitrato de plata acuoso, es decir, cada catión $\mathrm{Ag}^{+}$debe ganar un electrón para convertirse en $\mathrm{Ag}^{0}$. El agente encargado de donar el electrón es el ácido ascórbico, el cual será activado por el hidróxido de sodio (Tang et al., 2013b). Si la mezcla de las soluciones de nitrato de plata y ácido ascórbico se realizara directamente, podría dar lugar al crecimiento de partículas de plata con tamaño mayor al orden de los nanómetros; con el propósito de evitar esto, se utiliza la solución de citrato, que se encargará de formar una cobertura para evitar su coalescencia e inhibir su crecimietno; esto se conoce como pasivación. Los pasivantes son compuestos químicos que se unen a la superficie de las nanopartículas evitando la coalescencia (Caponetti, Pedone, Chillura Martino, Pantò y Turco Liveri, 2003).

El método propuesto se ha diseñado mediante la modificación de diversos métodos descritos en la literatura (Frank et al., 2010) y se compone de los siguientes pasos:

1. Con los reactivos descritos se prepararon las siguientes soluciones: ácido as-

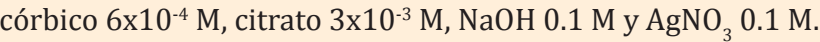

2. Se etiquetaron 3 vasos de precipitados.

3. A cada uno se añadieron $4 \mathrm{~mL}$ de la solución de ácido ascórbico y $4 \mathrm{~mL}$ de la solución de citrato.

4. Posteriormente, se ajustó el pH a cada mezcla con los valores de 10.9, 11.5 y 12.0 con la solución de $\mathrm{NaOH} 0.1 \mathrm{M}$. En este punto, los interesados pueden utilizar diversos valores de $\mathrm{pH}$ entre 8.0 y 12.0, teniendo cuidado de registrar el valor exacto para después compararlo con el color de las nanopartículas obtenidas.

5. Finalmente, se añadió $1.0 \mathrm{~mL}$ de la solución de nitrato de plata a cada vaso y las soluciones se dejaron en reposo. Después de un minuto comenzó la aparición de color.

Los centros de enseñanza que cuenten con técnicas de caracterización de sólidos podrán separar las nanopartículas de la solución mediante secado. En el presente trabajo, las soluciones se introdujeron en una estufa a $100{ }^{\circ} \mathrm{C}$ hasta obtener un polvo oscuro.

\section{Resultados}

Se realizaron tres ensayos de reducción de la sal de plata a pH 10.9, 11.5 y 12.0. Las soluciones se evaporaron y el polvo sólido se analizó por difracción de rayos X. El difractograma (figura 3) muestra una serie de reflexiones correspondientes a cristales de plata metálica registrada en la ficha 040783 de la base de datos del International Crystallography Centre Database (línea roja) y aparece con mezcla de nitrato de plata que no fue reducido durante la reacción (línea verde del difractograma). 
FIGURA 3. Difractograma de rayos $\mathrm{X}$ de las nanopartículas de los polvos obtenidos.

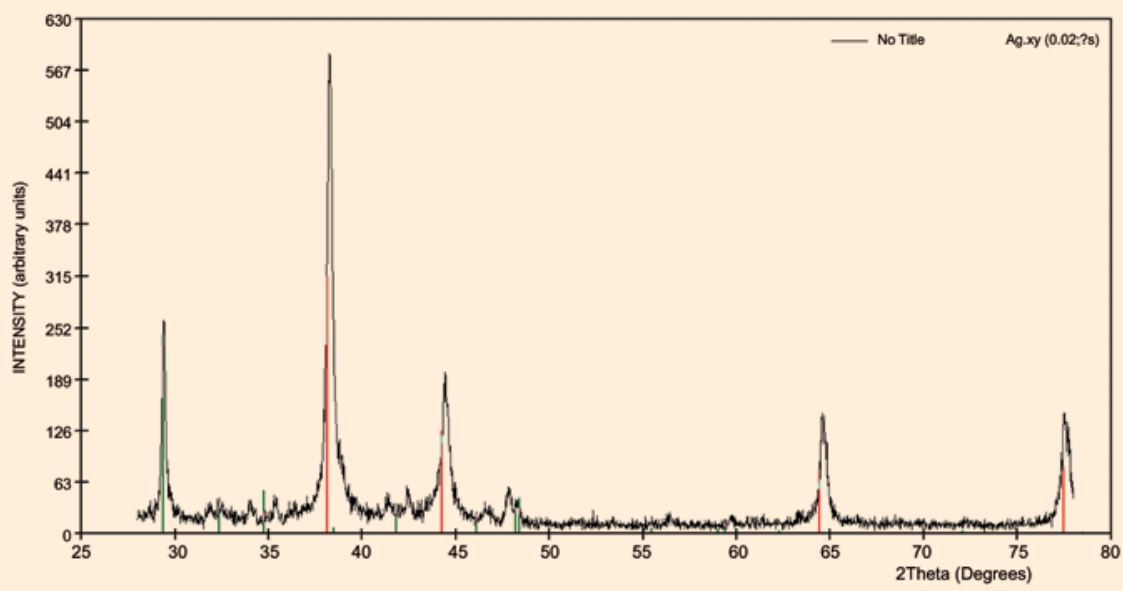

En la figura 4 se observan dos de las tres muestras de nanopartículas de plata. En la cual se tiene el frasco etiquetado como $\mathrm{pH}=\mathrm{B}$, que corresponde a un $\mathrm{pH}$ de 10.9, que presentó un color amarillo pálido que fue estable por al menos 3 días. Esta muestra adquiere dicho color debido al fenómeno de SPR.

$\mathrm{Al}$ aumentar el pH a 11.5 (muestra etiquetada como $\mathrm{pH}=\mathrm{A}$ en la figura 4), la solución de la plata se oscurece, pero se mantiene el color amarillo.

Retomando las imágenes obtenidas de la bibliografía (figura 2) la nanopartículas que presentan color amarillo presentan la SPR cerca de los $450 \mathrm{~nm}$. En esa misma figura, se muestran diversos colores, resultantes de diferentes tamaños de partícula. En los resultados obtenidos en el presente trabajo (figura 4), el color presentado

FIGURA 4. Ensayos realizados a distintos valores de $\mathrm{pH}(A=11.5$ y $B=10.9)$. Nótese la diferencia en la coloración.

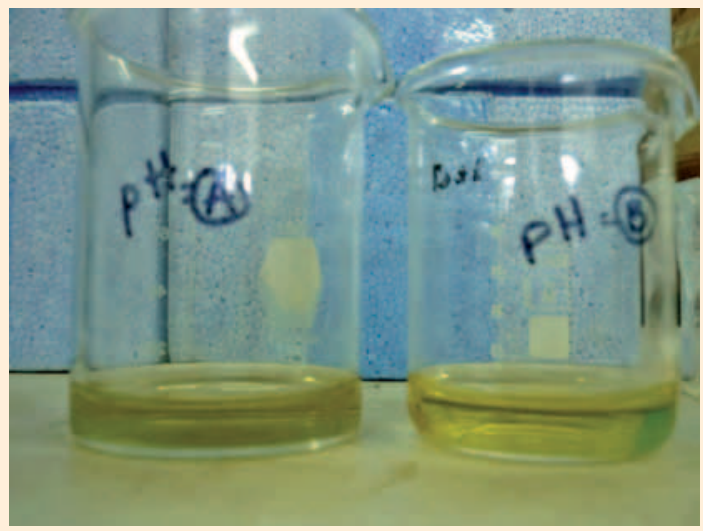


FIGURA 5. Comparación visual de los tres ensayos realizados denotando la diferencia en la concentración de nanopartículas de plata en la solución.

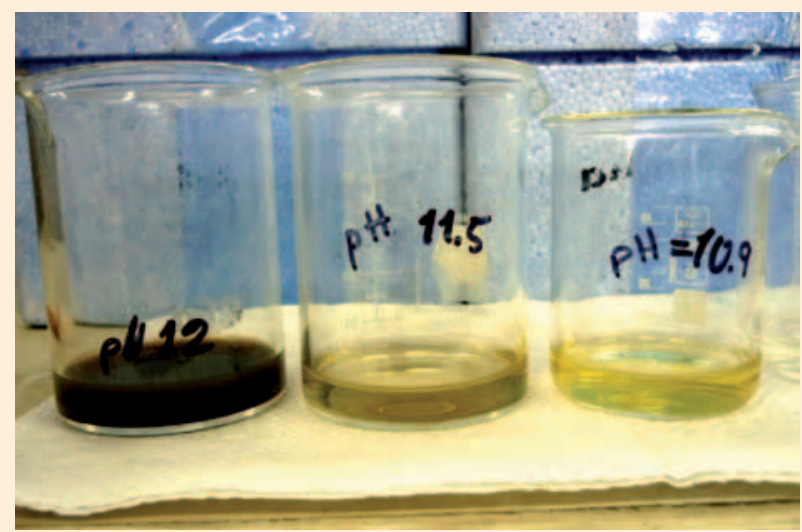

está en la gama del amarillo, lo cual sugiere que no hubo cambio en el tamaño de las partículas.

Una explicación al hecho que las muestras obtenidas sólo estén dentro de la región del amarillo es que el tamaño formado es el mismo a diferentes valores de $\mathrm{pH}$. Para sustentar esto, obsérvese la figura 6 que se reporta en la literatura, donde se logra apreciar la secuencia de tres muestras a diferentes valores de $\mathrm{pH}$. Percíbase que el único cambio es la tonalidad del color, siendo este resultado similar a un trabajo reportado anteriormente donde se demuestra que el cambio de tonalidad es debido a un cambio de concentración como se demuestra en la figura 6 (Chhatre et al., 2012).

Por lo tanto, con el método de síntesis propuesto en este trabajo se logró obtener nanopartículas de plata con un tamaño uniforme, y el único efecto del pH es el de aumentar la concentración de nanoparticulas.

Se sugiere a los interesados en utilizar el método diseñado modificar la cantidad de la solución de ácido ascórbico añadido para tener una diferente concentración de moléculas pasivante y promover la formación de otros tamaños de partícula.

El procedimiento diseñado para esta práctica utiliza reactivos de muy fácil acceso para cualquier laboratorio de educación media y superior, y además los residuos pueden ser completamente manejables, pues las soluciones de ácido ascórbico y cí-

FIGURA 6. Efecto de la concentración de nanoparticulas de plata de un mismo radio sobre el color. Las concentraciones son $47.7,42.9,33.4,28.6,23.9,21.5,19.1,16.7,14.3,13.1,11.9,9.5,7.2$ y $4.8 \mu \mathrm{g} / \mathrm{m}^{3}$, de izquierda a derecha. Imagen tomada de Chhatre y colaboradores (Chhatre et al., 2012).

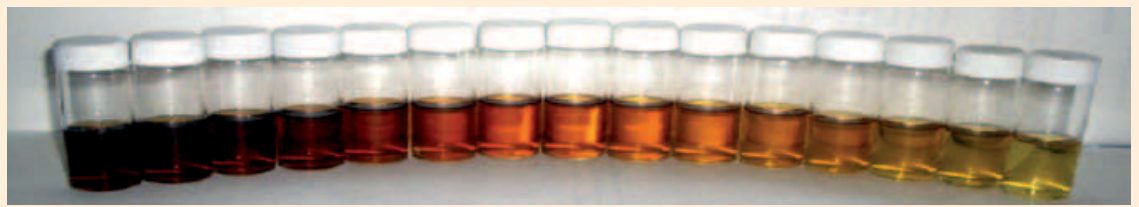


trico son completamente biodegradables si se descartan por el drenaje, mientras que las soluciones con nanopartículas de plata se pueden reciclar mediante una evaporación para retirar el agua, seguida por una calcinación para eliminar residuos de materia orgánica y una redisolución de las nanopartículas con ácido nítrico para retornar finalmente a la solución de nitrato de plata.

\section{Conclusiones}

Se ha propuesto una estrategia de síntesis nueva adaptada a partir de diversas fuentes bibliográficas. La obtención de las nanopartículas de plata fue exitosa por este método y permitió observar el color amarillo de la plata que es una diferencia contrastante con el color conocido en tamaño macrométrico. El método es sencillo y fácilmente puede ser desarrollado en cualquier laboratorio de enseñanza media y superior orientado al área de nanociencias ya que se utilizan reactivos de fácil acceso, baja toxicidad y de bajo costo.

A pesar de esta sencillez, los fundamentos técnicos y científicos que explican el comportamiento tan complejo de los materiales a escala nanométrica son novedosos y extensos. La demostración del efecto de resonancia del plasmón de superficie es uno de ellos y puede motivar a los estudiantes a profundizar en el estudio del comportamiento de la materia en escala nanométrica.

Se invita a los lectores interesados a reproducir este método variando más parámetros como $\mathrm{pH}$ y concentraciones del ácido cítrico y ascórbico para obtener otras posibles coloraciones.

\section{Agradecimientos}

Los autores agradecen al Conacyt el apoyo con las becas No. 273963, 286569 y 274013. A los encargados del Laboratorio de Electroquímica de la Universidad de Guadalajara, por facilitarnos algunos reactivos.

\section{RefERENCIAS}

An, W.; Zhu, T. y Zhu, Q. (2013) “Numerical investigation of radiative properties and surface plasmon resonance of silver nanorod dimers on a substrate". Journal of Quantitative Spectroscopy and Radiative Transfer, 1-8. doi:10.1016/j.jqsrt.2013.01.013.

Caponetti, E.; Pedone, L.; Chillura Martino, D.; Pantò, V. y Turco Liveri, V. (2003) "Synthesis, size control, and passivation of CdS nanoparticles in water/AOT/n-heptane microemulsions". Materials Science and Engineering: C, 23(4), 531-539. doi:10.1016/ S0928-4931(03)00030-4.

Chhatre, A.; Solasa, P.; Sakle, S.; Thaokar, R. \& Mehra, A. (2012) “Color and surface plasmon effects in nanoparticle systems: Case of silver nanoparticles prepared by microemulsion route". Colloids and Surfaces A: Physicochemical and Engineering Aspects, 404, 83-92. doi:10.1016/j.colsurfa.2012.04.016

Frank, A. J.; Cathcart, N.; Maly, K. E. y Kitaev, V. (2010) “Synthesis of silver nanoprisms with variable size and investigation of their optical properties: a first-year undergradua- 
te experiment exploring plasmonic nanoparticles". Journal of Chemical Education, 87(10), 1098-1101. doi:10.1021/ed100166g.

Tang, B.; Li, J.; Hou, X.; Afrin, T.; Sun, L. y Wang, X. (2013) “Colorful and antibacterial silk fiber from anisotropic silver nanoparticles". Industrial \& Engineering Chemistry Research, 52(12), 4556-4563. doi:10.1021/ie3033872.

Vankar, P. S. y Shukla, D. (2011) "Biosynthesis of silver nanoparticles using lemon leaves extract and its application for antimicrobial finish on fabric". Applied Nanoscience, 2(2), 163-168. doi:10.1007/s13204-011-0051-y.

Vasileva, P.; Donkova, B.; Karadjova, I. y Dushkin, C. (2011) “Synthesis of starch-stabilized silver nanoparticles and their application as a surface plasmon resonance-based sensor of hydrogen peroxide". Colloids and Surfaces A: Physicochemical and Engineering Aspects, 382(1-3), 203-210. doi:10.1016/j.colsurfa.2010.11.060. 